\title{
Single dopants in semiconductors
}

\author{
Steven R. Schofield \\ London Centre for Nanotechnology, and \\ Department of Physics and Astronomy \\ University College London \\ London, WC1H 0AH, UK
}

\section{Sven Rogge}

Centre for Quantum Computation and Communication Technology

School of Physics

The University of New South Wales

Sydney, NSW 2052, Australia

The controlled introduction of dopant atoms to semiconducting host materials is the corner stone of electronic device fabrication. Dopant atoms provide a means to modulate the electronic, optical, and magnetic properties of semiconductors [1], and it is now possible to control dopant profiles with true atomic-scale precision in the laboratory [2]. Moreover, industrial fabrication methods are now capable of producing features with sub-10 nm precision [3] which therefore contain only a small numbers of dopants. This extraordinary control of dopant atom placement and semiconductor feature patterning provides exciting possibilities for the creation of quantum (opto)electronic devices including devices for quantum information processing. Furthermore, it can be argued that building such devices in semiconductor hosts provides a more straightforward route for their incorporation with conventional semiconductor electronics compared with competing quantum device architectures. At the same time there are new challenges for conventional devices at these length scales; the very small number of dopants involved in sub-10 nm structures means the position of individual dopants has a dramatic effect on charge transport due to the effects of scattering, quantisation, and tunnelling [4]. The present challenge is to develop a deeper understanding of the physics underpinning small numbers of dopant atoms in device-relevant atomic-scale architectures. This will necessarily include the further development of methods for fabricating such structures and the refinement of the theoretical tools appropriate for modelling them.

This special edition showcases recent developments in the study of single dopants in semiconductors. As might be expected in a field that inherently sits at the confluence of "top-down" and "bottom-up" nanoscience approaches [5, 6], the papers cover a range of experimental techniques, including scanning tunnelling microscopy and 
spectroscopy (STM/STS) [7, 8, 9], single-ion ion implantation [10], charge transport spectroscopy [11], and electrical spin readout [12]. There are also theoretical investigations utilising atomistic tight binding [13] and central cell corrected effective mass theory [14].

Numerous contributions in this special edition focus on dopants in silicon. This can be attributed to the central role of silicon in microelectronic devices and the recent interest in single dopant quantum devices inspired by the Kane quantum computer proposal [15]. Correspondingly, phosphorus (in silicon) features heavily, as do the other $n$-type dopants arsenic, antimony, and bismuth. Muhonen et al. [11] report measurements that build upon their recent demonstrations of coherent control and single-shot readout of the electron and nuclear spins of single phosphorus atoms. They report gate fidelities upward of $99.95 \%$ for phosphorus in isotopically enriched silicon 28, which is sufficient for certain error correction protocols and thus bodes well for donor-based quantum computing. Jehl et al. [12] fabricate devices with gated pairs of dopants (phosphorus or arsenic) coupled to source-drain electrodes that they term a coupled atom transistor. These are formed statistically or via ion implantation into silicon nanowires, and they demonstrate the preservation of orbital quantisation of the donors in these structures and measure their charge coherence times. To fabricate more complex structures via ion implantation it is necessary to develop new pathways for deterministic ion implantation at the single- and few-ion level; this is explored by Van Donkelaar et al. [10], who in addition to the above mentioned $n$-type dopants in silicon also investigate the implantation of nitrogen into diamond.

The study of individual dopant atoms with STM/STS has historically focussed on GaAs due to the relative ease of in-situ sample cleaving that produces a surface free of surface states and provides ready access to substitutional dopants. Correspondingly two of the STM papers report investigations of dopants in GaAs. Smackman et al. demonstrate the ability to control the charge state of Si dopants in the GaAs(110) surface layer using both the electric field of the STM tip and optical illumination [7]. Lee et al. [8] report an STS investigation of Mn deep level acceptor states and how their energetics are influenced by nearby charged defects. The STM contribution from Voisin et al. [9] reports low temperature donor single-electron transport spectroscopy of arsenic in silicon, where the STM tip forms one of the source-drain electrodes and the degenerately-doped substrate the other. The donor itself is isolated by its location within a donor-depletion region at the sample surface, and by the tunable vacuum tunnel barrier.

A recurring theme throughout the issue is the need to extend, or develop new theories 
to model these systems. For example, Voisin et al. [9] apply the theory of charge transport in mesoscale systems to the single atom level, and Lee et al. [8] posit that the understanding of donor spectroscopy by STM is still in its infancy, despite that semiconductors have been explored with STM since the invention of the technique. The two dedicated theory papers make strides toward robust theoretical descriptions of donor devices. Usman et al. [13] perform atomistic tight-binding simulations of arsenic in silicon, including both non-static dielectric screening and the effect of lattice strain - important parameters in any nanoscale device architecture - to find significantly improved agreement with experiment. Saraiva et al. [14] produce a detailed review for modelling silicon nano-devices with one or two group $\mathrm{V}$ donors using effective mass theory, including in their calculations the important effects of charge occupancy of the donors. They also discuss the validity and limitations of the several commonly employed analytical approximations.

This is a very exciting time in the development of both the science and the engineering of atomic-scale dopant structures and devices in semiconductors. We hope that you enjoy reading these articles as much as we have, and we greatly thank all the contributors to this special issue.

\section{References}

[1] P. M. Koenraad and M. E. Flatté, Nature Mater., 10, 91 (2011).

[2] M. Fuechsle, J. A. Miwa, et al., Nature Nano., 7, 242 (2012).

[3] I. Ferain, C. a. Colinge, and J.-P. Colinge, Nature, 479, 310 (2011).

[4] V. P. Georgiev, E. A. Towie, and A. Asenov, IEEE Trans. Elec. Dev., 60, 965 (2013).

[5] J. Tucker and T.-C. Shen, Solid-State Electronics, 42, 1061 (1998).

[6] M. Lundstrom, Science, 299, 210 (2003).

[7] E. P. Smakman, et al., J. Phys.: Condens. Matter, ?, ? (2015).

[8] D. Lee, et al., J. Phys.: Condens. Matter, ?, ? (2015).

[9] B. Voisin, et al., J. Phys.: Condens. Matter, ?, ? (2015).

[10] J. A. van Donkelaar, et al., J. Phys.: Condens. Matter, ?, ? (2015).

[11] J. T. Muhonen, et al., J. Phys.: Condens. Matter, ?, ? (2015). 
[12] X. Jehl, et al., J. Phys.: Condens. Matter, ?, ? (2015).

[13] M. Usman, et al., J. Phys.: Condens. Matter, ?, ? (2015).

[14] A. L. Saraiva, et al., J. Phys.: Condens. Matter, ?, ? (2015).

[15] B. E. Kane, Nature, 393, 133 (1998). 